Revue d'histoire de l'Amérique française

RAS REVUE D.HISTOIRE DE L'AMÉRIQUE FRANÇAISE

\title{
Le bicaméralisme québécois : rétrospective comparative
}

\section{Edmond Orban}

Volume 25, numéro 2, septembre 1971

URI : https://id.erudit.org/iderudit/303064ar

DOI : https://doi.org/10.7202/303064ar

Aller au sommaire du numéro

Éditeur(s)

Institut d'histoire de l'Amérique française

ISSN

0035-2357 (imprimé)

1492-1383 (numérique)

Découvrir la revue

Citer cet article

Orban, E. (1971). Le bicaméralisme québécois : rétrospective comparative.

Revue d'histoire de l'Amérique française, 25(2), 191-203.

https://doi.org/10.7202/303064ar d'utilisation que vous pouvez consulter en ligne.

https://apropos.erudit.org/fr/usagers/politique-dutilisation/ 


\title{
LE BICAMÉRALISME QUÉBÉCOIS : RÉTROSPECTIVE COMPARATIVE
}

\author{
Edmond Orban \\ professeur agrégé \\ Université de Montréal
}

Il y a à peine deux ans, le Conseil législatif de Québec a été aboli. C'est un événement plus important qu'il n'y paraît à première vue. Pour mieux en juger, nous essaierons de situer l'action passée de cette Chambre législative par rapport à l'évolution en cours dans d'autres pays. Dans la première partie de cette rétrospective, nous effectuerons un rapide survol des Chambres hautes au niveau local (Länder, Etats, provinces, etc...) et central. Au niveau local ${ }^{1}$, nous verrons que le bicaméralisme est devenu une exception. Par contre, au palier supérieur, Congrès américain, parlements divers, etc..., on lui trouve encore des raisons d'être importantes.

Dans une seconde partie, nous essaierons de voir si le type d'argument invoqué plus haut, peut s'appliquer aux Chambres hautes locales. Ce type d'approche nous permet de tirer des conclusions plus nuancées à propos d'une expérience passée. Elle nous montre en quoi le Conseil législatif correspond à certaines tendances qu'on peut observer à divers degrés ailleurs. Mais elle fait ressortir d'autant plus nettement certaines caractéristiques complexes plus spécifiquement québécoises.

\section{I - Chambres HAUTES LOCALES ET CENTRALES}

1. Situation du bicaméralisme local en général

Il n'y a plus guère que dans les Etats fédéraux que l'on retrouve encore deux Chambres législatives au niveau local. Et encore, seuls quelques-uns parmi ceux-ci ont créé ou maintenu ce système. En 1949, en Allemagne fédérale, malgré l'importance de certains Länder, on n'a pas cru bon de l'instaurer. Si l'Etat du Vermont, avec un demi-million d'habitants, a créé une Chambre haute, on peut s'étonner à première vue que la RhénanieWestphalie (plus de 17 millions d'habitants) ait jugé inutile ce type d'institution. En réalité, on a estimé qu'une seule Chambre

1 Par local, nous excluons donc les autres paliers, notamment les gouvernements municipaux, qui d'ailleurs relèvent soit du gouvernement central, soit des provinces, Etats, etc...

[ 191 ]

RHAF, vol. 25, no 2 (septembre 1971) 
législative suffisait au niveau des Länder, d'autant plus que les institutions centrales (ou fédérales) ont été conçues de façon à protéger les droits des Länder. C'est notamment dans cette perspective que le Bundesrat a été institué sans parler d'autres mécanismes prévus au Bundestag, mode de scrutin, Cour suprême fédérale, etc...

A première vue, la Bavière semble constituer une exception importante. Son Sénat, de type plutôt corporatif, est composé de 60 membres élus pour six ans. Cette institution a des précédents dans l'histoire assez particulière de la Bavière. Mais ce n'est plus une véritable Chambre législative; la constitution bavaroise a réduit ses pouvoirs à un rôle purement consultatif. ${ }^{2}$ Le Sénat bavarois ne peut donc être comparé aux autres Chambres hautes que nous verrons plus loin.

En Suisse, le bicaméralisme au niveau des cantons n'existe pas. Les traditions décentralisatrices y sont cependant plus anciennes et plus solides que dans n'importe quelle autre fédération du monde. Les cantons, libres encore de l'instaurer s'ilis le désirent, ont un point de vue comparable à celui que nous venons de mentionner. Le Conseil des Etats, entre autres, (avec ses deux représentants par canton) fait parti aussi de l'arsenal de défense des cantons. Le tout est complété par le referendum et l'initiative populaire, système de protection dont se sont inspirés plusieurs Etats aux Etats-Unis.

Il ne s'agit pas ici d'étudier l'efficacité de ces moyens mais par opposition, ils nous aident à mieux situer les cas particuliers que constituent les bicaméralismes locaux, australiens, américains et canadiens. Dans ces trois cas, nous nous trouvons dans des régions qui, dans le passé du moins, ont été profondément marquées par le parlementarisme britannique. Pendant longtemps, ce dernier a été tour à tour un pôle d'attraction ou de répulsion, lorsque les constitutions locales ont été mises en place ou modifiées.

Aux Etats-Unis ces mouvements contradictoires sont particulièrement frappants. Le bicaméralisme local y trouve son origine dans certaines législatures coloniales. Celles-ci, tout comme

2 Voir la constitution bavaroise du 2 décembre 1946. L'article 34 spécifie que le Sénat de Bavière est la représentation des corps sociaux, économiques, culturels et communaux du Land. L'article 34 fixe cette composition à 60 sénateurs. Ces derniers sont pour la plupart élus par des corps intermédiaires: représentants de l'industrie et du commerce, représentants de l'agriculture, syndicats, professions libérales, universités, communes, confessions religieuses, etc. 
dans plusieurs provinces canadiennes, étaient plus ou moins imposées par le colonisateur. Les membres de ces Chambres hautes étaient choisis parmi les propriétaires les mieux nantis, sur recommandation du gouverneur (représentant du gouvernement de Londres). Elles étaient destinées à freiner, voire à paralyser l'Assemblée élue. Par contre, quand les colonies américaines devinrent indépendantes (entre 1776 et 1781), trois d'entre elles adoptèrent le monocaméralisme: le Vermont, la Pennsylvanie et la Georgie. D'autre part, un peu plus tard la convention de Philadelphie, en 1787, allait créer une Chambre haute élue par les Etats membres, très différente par rapport à la Chambre des Lords. Ceci aura exercé une influence considérable en ce qui concerne la survivance, sous d'autres formes, du bicaméralisme local. Finalement, les trois Etats mentionnés plus haut vont adopter à leur tour le bicaméralisme et, à partir de 1836, tous les Etats sans exception ont une seconde Chambre, avec cette différence qu'on ne s'inspire pas du modèle londonien, puisque toutes ces Chambres sont élues.

$\mathrm{Au}$ Canada, en 1867, les provinces constituent une fédération qui n'est pas encore indépendante du gouvernement de Londres. Celles qui, à cette époque, optent pour le bicaméralisme, choisissent ou maintiennent le système de la Chambre haute nommée par le gouvernement provincial. Au Québec pourtant, sous l'influence d'un mouvement nationaliste dirigé par Papineau, le gouvernement colonial avait toléré antérieurement le principe de l'élection pour le Conseil législatif. Cette expérience amorcée à partir de 1856 fut définitivement arrêtée en 1867. Et pourtant, comme l'a écrit Jean-Charles Bonenfant, "Jamais Chambre haute canadienne n'eut plus de prestige." ${ }^{3}$ Quoi qu'il en soit, il est intéressant de noter ici que les partisans du Conseil législatif élu se tournaient vers les Etats-Unis pour y chercher un modèle sur ce point-là du moins. C'est ce que firent également les Etats australiens en optant pour une Chambre haute élue.

En réimposant, en 1867 , le principe de la nomination des conseillers législatifs québécois, on allait à contre-courant par rapport à l'évolution observée aux Etats-Unis. ${ }^{4} \mathrm{~A}$ certains moments, ce facteur pèsera lourdement dans la balance politique au Québec, comme l'avait prévu un leader libéral en 1864:

3 J.-Ch. Bonenfant, "Le bicaméralisme dans le Québec", Canadian Journal of Economic and Political Science (1963), XXIX: 503.'

${ }^{4}$ L'Acte de l'Amérique du Nord britannique de 1867 comporte, à l'article 72 , une clause précisant ce mode de nomination à vie par le lieutenantgouverneur au nom de la Reine. 
Il n'est pas difficile de prévoir l'effet que pourra avoir sur le fonctionnement de la constitution, un Conseil législatif composé d'un nombre fixe de membres nommés à vie par la Couronne. Nous savons par expérience, le pouvoir qu'un semblable corps pourrait exercer pour entraver les vœux de l'opinion publique et paralyser toute législation progressive ${ }^{5}$.

La suppression du Conseil législatif de Québec en 1968 constitue donc un événement important pour l'évolution du bicaméralisme local dans les trois pays mentionnés. Aux Etats-Unis, en effet, tous les Etats ont gardé leur Chambre haute; il faut remonter jusqu'en 1937 pour voir la première et dernière abolition d'un sénat local (Nebraska). En Australie, la seule abolition opérée remonte à 1924. Au Canada la situation se présente très différemment. Tout d'abord la province la plus importante, l'Ontario et d'autres provinces de l'Ouest ont jugé ce système inutile. Le Manitoba a eu une Chambre haute nommée entre 1870 et 1876, l'Ile-du-Prince-Edouard a supprimé la sienne en 1893 presqu'en même temps que le Nouveau-Brunswick, son voisin. Enfin, la Nouvelle-Ecosse, provinec plutôt traditionaliste, réussit à abolir son Conseil législatif ${ }^{6}$ en 1928. C'était le plus ancien du Canada et cette opération fut réalisée avec difficulté. Quant au Conseil législatif de Québec, il sera la dernière Chambre haute locale au Canada qui réussira à survivre jusqu'en $1968^{~}$.

\section{Perspectives, en fonction du bicaméralisme central}

Le bicaméralisme local a évolué en fonction du bicaméralisme central, le modèle étant constitué par la Chambre des Lords ${ }^{8}$, ensuite par le Sénat américain ou par une combinaison d'éléments inspirés par ces deux prototypes. Cependant au niveau supérieur, le premier modèle, la Chambre des Lords, tend à disparaître; les "Parliament Acts" de 1911 et 1949 ont virtuellement complété l'amputation de ses pouvoirs. Par contre, les Chambres élues continuent à subsister dans de nombreux pays malgré une opposition croissante et gardent des pouvoirs importants. Dans les Etats fédéraux, la raison majeure invoquée pour maintenir une Chambre haute est la défense des intérêts locaux.

5 Manifeste d'Antoine-Aimé Dorion, rédigé le 7 novembre 1864.

6 J. S. Bourinot, "The constitution of the Legislative Council of Nova Scotia", in Transactions of the Royal Society of Canada (Ottawa, 1936) : 141-173.

7 Pour l'Afrique du Sud (Cap Bonne-Espérance, Natal, Orange, Transvaal) la question a été réglée depuis longtemps, voir : Keith, Responsible Government in the Dominions (Oxford, 1912), chap. VII.

8 Jusqu'en 1968, les Règles et Règlement du Conseil législatif de Québec, comportaient encore ce qui suit à l'article 92: "Dans tous les cas imprévus, on doit suivre les règles, formes et usages de la Chambre des Lords." 
Nous n'avons pas à mesurer ici la portée de cet argument. Il n'empêche que la plupart des observateurs estiment qu'à des degrés divers, le Bundesrat allemand, le Conseil des Etats en Suisse, le Sénat américain jouent un rôle sur ce plan. Parlant d'une façon générale, Wheare a écrit avec raison:

There is a fear that the executive and legislature depending primarily on numbers, may adopt policies which might be opposed but ineffectually opposed by the less popular states which are less strongly represented in the lower house 9 .

Ce type de Chambre constitue donc un contrepoids important pour l'équilibre des Etats fédéraux. Il doit permettre de défendre et aussi de canaliser les forces centrifuges qui existent partout. Cette constatation ne semble cependant pas s'appliquer aux sénateurs canadiens qui sont nommés à vie par le gouvernement central (contrairement au Sénat australien où chaque Etat élit dix sénateurs pour six ans). Malgré l'obligation qu'ont les gouvernements de respecter un certain quota par province, le Sénat canadien n'a pas été un obstacle sérieux pour les tendances centralisatrices du gouvernement canadien exprimées aux dépens parfois de l'autonomie des provinces. Un spécialiste américain du fédéralisme a écrit ce qui suit à ce sujet: "The senators never have had a sense of representing provinces and the canadian Senate has never been regarded as a bulwark of states' rights." 10 A l'origine, la première Chambre haute, la Chambre des Lords ne s'est pas développée dans ce but. Sa fonction première était de représenter et de défendre une classe sociale supérieure. $\mathrm{Au}$ fur et à mesure que la Chambre basse a pris de l'importance, la Chambre haute a progressivement perdu de l'initiative pour se cantonner de plus en plus dans un rôle de frein. Il y a une corrélation en sens inverse entre la montée de la Chambre élue et le déclin de la Chambre nommée. Ce mouvement exprime les transformations de la société anglaise, mais l'une de ses caractéristiques est précisément l'élargissement progressif du mode de scrutin. En d'autres termes une Chambre législative nommée semble de plus en plus condamnée. John Stuart Mill écrivait déjà ce qui suit au dix-neuvième siècle:

If there are two Houses, one considered to represent the people, the other to represent only a class, or not to be

${ }^{9}$ R. C. Wheare, Federal Government (Londres, 1968), 189.

10 W. H. Riker, Federalism (New York, 1964), 117. Ce point de vue a été exprimé avec différentes nuances par deux professeurs canadiens qui, par ailleurs, se dissocient complètement quant aux réformes à apporter au Sénat canadien pour lui faire jouer le rôle en question. Voir: R. A. Mackay, The unreformed Senate of Canada (Carleton, 1963), 112-123 Jacques-Yvan Morin, "Réforme du Sénat", Cité Libre (1964), n 68: 4-7. 
representative at all, I cannot think that where democracy is the ruling power in society, the second House would have any real ability to resist even the aberrations of the first 11 .

Les partisans du bicaméralisme ont compris que, pour survivre, les Chambres hautes devaient reposer avant tout sur le principe de l'élection.

On observe un grand nombre de procédés quant au mode de scrutin. Il peut être plus ou moins direct, de façon à représenter davantage les milieux ruraux et les petites villes par exemple. C'est un peu le cas du Sénat français sous la troisième et la quatrième République. En Belgique, malgré un mode de scrutin tempéré par d'autres éléments, le Sénat est devenu une réplique de la Chambre basse au point de vue de la composition politique et sociale. ${ }^{12}$ Le même phénomène semble s'opérer en Italie. Parlant du Sénat italien, Maranini a écrit à ce sujet:

Son défaut réside dans l'identité de composition des deux chambres et tant que celle-ci subsistera, le système se bornera à rendre plus longues et plus laborieuses les procédures légisiatives. Ce bicaméralisme ne constitue donc pas un obstacle sérieux à la concentration du pouvoir ni à la dictature parlementaire ${ }^{13}$.

Le danger qui menace donc ce type de Chambre n'est pas d'être considérée comme "réactionnaire" ou non représentative; il réside plutôt dans le fait que ces Chambres hautes sont inutiles, à moins qu'elles ne se réforment de façon à défendre les intérêts régionaux. C'est ce que souligne Maranini, rejoignant ainsi l'argument mentionné au début de ce paragraphe. Frein anti-démocratique ou double emploi, tel semble être le dilemme énoncé par l'abbé Sieyès dans "Qu'est-ce que le Tiers Etat?"

A quoi bon deux Chambres? Si elles sont d'accord, il y en a une d'inutile; si elles sont divisées, il y en a une qui non seulement ne représente pas la volonté du peuple mais qui l'empêche de prévaloir, c'est la confiscation de la souveraineté populaire.

Il reste quand même, dans ce cas, une fonction non négligeable, celle de retarder le passage de projets de loi hâtivement rédigés. Constatant qu'en Suède on retrouve les mêmes majorités

11 John Stuart Mill, Considerations on representative Government (New York, 1962), 327.

${ }_{12} \mathrm{Au}$ sujet de cet aspect du bicaméralisme en Belgique, voir $\mathrm{Ch}$. Goossens, "Le bicaméralisme en Belgique", Revue internationale de sciences administratives (Bruxelles, 1951), n² 2. - Fusilier, Les Monarchies Parlementaires (Paris, 1960), 469-489.

13 Maranini, L'Italie (Paris, 1961), 56. 
dans les deux Chambres depuis cinquante ans, un observateur ${ }^{14}$ suédois a clairement démontré que, dans son pays, la Chambre haute n'avait plus pour fonction de représenter certains intérêts économiques et sociaux. Son rôle est très limité. Elle demeure toutefois une garantie contre la législation hâtive, mal faite au point de vue technique.

En l'occurrence, la Chambre haute devrait être composée d'experts de talent, mais on se heurtera toujours au problème de l'élection et des pouvoirs à octroyer. S'il s'agit uniquement d'améliorer techniquement les projets de loi, il existe d'autres procédures et moyens de consultation de nature à résoudre ce problème.

En conclusion la position de la Chambre haute même élue est devenue de plus en plus précaire dans le monde. En Suède, elle ne subsiste qu'à grand-peine, la Finlande, la Nouvelle-Zélande et le Danemark l'ont abolie de même que plusieurs autres pays. Ailleurs, on essaye de la réformer de toutes sortes de façons. Les résultats ont au moins un point commun, ils vont dans le sens d'une réduction des pouvoirs, qui souvent est une transition lente mais irréversible vers l'abolition. Quant aux nouveaux Etats, que ce soit au Moyen-Orient, dans le Sud-Est asiatique ou en Afrique, ils ont opté sans guère d'hésitation pour le monocaméralisme. Les rares exceptions se retrouvent précisément dans l'un ou l'autre pays, tel le Congo Kinshasa où certains dirigeants tentent ou ont tenté d'implanter le fédéralisme avec les difficultés que l'on sait. Ce qui signifie que, même au niveau du gouvernement central, une Chambre haute représentant les Etats membres ne s'impose plus nécessairement. S'il fallait résumer à l'extrême, nous dirions que les Chambres hautes ont tendance à se maintenir dans les Etats fédéraux alors qu'elles sont de plus en plus menacées dans les Etats unitaires. Mais cette distinction est un peu illusoire, car il est parfois très difficile de distinguer un gouvernement fédéral d'un gouvernement très décentralisé, même si ce dernier porte une étiquette différente.

Il n'en reste pas moins vrai que l'argument fédéraliste s'avère le plus solide pour justifier une seconde Chambre. Mais dans ce cas il faut que le fédéralisme ne soit pas trop atteint par la centralisation au palier supérieur du gouvernement. Faute de quoi, cette seconde Chambre, quels que soient sa composition et ses pouvoirs, risque d'être inutile.

\section{II - DÉClin DU BICAMÉRALISME LOCAL : ETATS-UNIS, AUSTRALIE, QUÉBEC}

14 E. Hästad, The Parliament of Sweden (Londres, 1957), 51-61. 
1. Incidences de la composition de la Chambre haute.

Les Chambres hautes locales des Etats membres d'une fédération font partie du gouvernement local; elles n'ont donc pas la possibilité d'invoquer les arguments apportés en faveur du Bundesrat allemand ou des Sénats américain et australien par exemple. Autrement dit, elles se trouvent dans une situation comparable à celle des sénats des Etats souverains unitaires. Mais là encore, leur position est comparativement plus faible car les provinces et Etats membres des Fédérations sont situés à un palier inférieur de gouvernement par rapport au gouvernement central des fédérations actuelles.

Il est vrai que certains Etats, tels la Californie, New-York ou même l'Etat de Nouvelle-Galles du Sud en Australie, constituent des entités économiques plus puissantes que beaucoup d'Etats jouissant juridiquement d'un statut d'Etat souverain. Cette réserve faite, nous pouvons présumer que le danger qui menace le plus ces sénats locaux est précisément celui de double cmploi; et ici on rejoindrait alors assez logiquement les constatations faites dans les pays souverains, dits unitaires. C'est tout d'abord du côté de la composition que cette hypothèse se vérifie généralement. Il y a très peu de différence au point de vue de la représentation des groupes sociaux dans les deux Chambres au niveau des Etats américains. Parfois la durée du mandat est plus longue, l'âge moyen des sénateurs plus élevé, leur nombre plus réduit, mais la composition varie très peu d'une Chambre à l'autre. La situation n'a guère changé alors qu'il y a déjà trente ans un observateur américain écrivait ce qui suit:

Today, there is little distinction between the two houses.

More often than not, their members are elected from the same districts, by the same people and at the same election, so that there is little ground for jealousy or rivalry between them. Where the houses differ in point of view today it is a matter not of social position but of political partisanship ${ }^{15}$.

D'autre part, ce qui peut compliquer la tâche des Chambres, c'est le fait que les majorités peuvent être de partis opposés, comme on le voit au Congrès national. En cas de conflit entre les deux Chambres locales, on adopte alors la procédure des conférences de comités. Les sessions s'y tiennent généralement à huis clos et les Chambres entérinent très souvent les quasi décisions prises à l'intérieur de ces comités restreints. C'est évidemment dans ce genre d'endroit que les groupes de pression ont le plus de chance d'exercer leur influence. Ce système, conséquence du

${ }^{15}$ A. W. Johnson, The unicameral legislature (The University Minnessota Press, 1938), 61. 
bicaméralisme, présente deux inconvénients: $1^{\circ}$, il permet d'éviter de donner une publicité à des débats et à des prises de décisions portant sur des projets de loi importants et controversés ; $2^{\circ}$, il permet au législateur de la Chambre basse d'échapper à ses responsabilités. En effet, il peut voter une loi progressive, en espérant que l'autre Chambre la refusera, ou vice versa, selon la conjoncture politique et sociale ${ }^{16}$. Ceci provoque toutes sortes de manœuvres dont les conférences de comités constituent le principal théâtre. Le dilemme énoncé par l'abbé Sieyès se pose aussi pour le bicaméralisme local, explicité en d'autres termes, mais avec la même logique un peu rigoureuse:

The case for two chambers as a guaranty of critical revision for legislation may be summarized in the assertion that if both houses are of the same political complexion, one chamber is superfluous, if the two houses are controlled by different parties, the second is likely to be obstructive ${ }^{17}$.

En résumé, si elles sont de même composition politique elles sont inutiles; dans le cas contraire l'une des deux risque de bloquer le système, s'il n'existe l'échappatoire prévu plus haut, avec tous les aléas qu'il présente. ${ }^{18} \mathrm{Au}$ Conseil législatif de Québec la conférence des commissions a existé aussi, mais comme elle s'inspirait du régime parlementaire anglais, cet organisme n'a pas du tout joué le même rôle, chacune des deux Chambres désirant garder intacts tous leurs pouvoirs de décision et limitant la portée des comités.

$\mathrm{Au}$ Québec, par contre, même si les deux Chambres étaient composées par une majorité du même parti, on a, à certaines périodes de l'histoire, enregistré des conflits entre les deux Chambres, précisément parce que leur composition sociale était différente.

Dans le cas des Etats australiens, à cet égard, on observe un Sénat qui s'apparente davantage à celui observé dans les Etats américains, avec cette différence qu'il est un peu hybride, s'inspirant à la fois du régime parlementaire anglais avec le principe

16 On retrouve cet inconvénient même dans un bicaméralisme aussi différent que celui qu'a connu la province de Québec. Voir, par exemple, E. Orban, "La fin du bicaméralisme au Québec", Canadian Journal of Political Science, Revue canadienne de Science politique (1969), no 3: 323.

17 A. W. Johnson, op. cit., 63.

18 Il est curieux de constater comment cette situation a peu ou pas changé au cours du vingtième siècle. Voir J. D. Barnett, "The bicameral system in state legislation", The American Political Science Review, IX (1915). 
de la responsabilité ministérielle, tout en empruntant certains éléments du Sénat américain.

La situation des Conseils législatifs de la Nouvelle-Ecosse et du Québec était beaucoup plus claire à ce point de vue parce qu'ils représentaient non pas seulement des partis, mais aussi des groupes sociaux différents à divers degrés.

\section{Activité matérielle}

Sur le plan de l'activité matérielle, on enregistre une tendance au déclin à peu près partout dans les Chambres hautes régionales. Les projets de loi sont de moins en moins introduits à la Chambre haute, au profit de la Chambre basse. Dans certains états de la Nouvelle-Angleterre comme le Vermont et le Maine, le pourcentage des "bills" présentés à la Chambre haute se situe autour de $15 \%$; idem en Ohio. On observe le même phénomène ailleurs, en dépit de certaines réformes qui n'enrayent pas cette tendance. Au Québec, l'introduction de projets de loi au Conseil législatif se chiffrait à environ $20 \%$ au début du vingtième siècle. Peu avant 1960, ce pourcentage était réduit à néant.

Les Etats australiens n'échappent pas à ce mouvement. Certains réformateurs avaient souvent suggéré que l'on fasse travailler davantage les Chambres hautes en y introduisant les projets de loi non controversés et les "bills" privés, de façon à épargner le temps des Chambres basses encombrées de travail (du moins dans les grands Etats). Le résultat fut décevant comme le signale une étude de Ken Turner:
And yet little advantage has been taken of this opportunity to initiate discussion of important public matters or non contentious bills. Indeed the extensive use of the "gag" in the assembly, has been accompanied by an apparent decline in the number of those seeking access to the legislative council and a clear decline in its assertion of initiative 19 .

Partout, ceci se traduit d'ailleurs par une diminution des journées de session, comparativement avec les Chambres basses. Dans plusieurs grands Etats australiens, les journées de travail ont diminué de près de la moitié, atteignant une moyenne souvent inférieure à trente par an (au Québec il y a eu vingt-deux séances en 1963). On pourrait au moins s'attendre à ce que ce temps soit utilisé rationnellement. Mais un phénomène commun à la majo-

19 Ken Turner, "The role of an Upper House: The Legislative Council of New South Wales", The Australian Journal of Politics and History, $\mathrm{XI}, \mathrm{n}^{\circ} 1$ (1965) : 49. 
rité des Législatures se retrouve ici également, à savoir l'accumulation des projets de loi en fin de session. Cette situation imputable surtout aux gouvernements n'est pas en soi liée au bicaméralisme, mais elle empêche la seconde Chambre d'exercer sa fonction de contrôle et de critique, d'autant plus que la majorité des projets de loi passent d'abord à la Chambre basse, comme nous l'avons indiqué plus haut.

Lorsqu'il était encore gouverneur de l'Etat de New York, Franklin Roosevelt a signalé à maintes reprises les dangers de ce "rush" des fins de session dans les législatures des Etats. Cette situation n'a guère été corrigée et elle enlève encore du poids à l'argument selon lequel la seconde Chambre permet d'apporter des délais, de retarder le passage de bills improvisés. Parlant du Conseil législatif de la Nouvelle-Galles du Sud, Ken Turner résume la situation comme suit:

The existence of a second chamber does not guarantee substantial delay; bills are regularly sent up late in the session and can be rushed through the Council too. In fact during the 1950 s about 30 per cent of bills dealt with in the Council were dismissed in one day, slightly more were dealt withon two separate days, etc ...20

$\mathrm{Au}$ Québec la situation était encore pire, le budget étant envoyé régulièrement dans les trois derniers jours de la session. Il est même arrivé que le budget soit présenté, discuté, voté et reçoive la sanction du lieutenant-gouverneur, au cours du dernier jour de la session. On voit mal comment évoquer l'argument délai de réflexion qui pour beaucoup restait la seule raison d'être du bicaméralisme local. ${ }^{21}$

\section{Réformes, force d'inertie et abolition récente}

Partout il y a eu des tentatives de réformes s'inspirant des essais réalisés à l'étranger, tant dans les Etats unitaires que fédéraux. On a essayé de transformer les Chambres hautes en Chambres d'experts, de représentants des différents groupes d'intérêt. Mais on s'est heurté à une sorte d'indifférence générale. Les groupes d'intérêts ont d'ailleurs d'autres moyens pour s'exprimer et se défendre (mass-média, action auprès des gouvernements et de l'administration, partis politiques, etc...).

$20 \mathrm{~K}$. Turner, op. cit., 47.

21 A ce sujet, pour une appréciation un peu sommaire sur les Sénats locaux aux Etats-Unis, voir J. F. Zimmerman, State and local government (New York, 1962), 96. 
On se demande si ce n'est pas un peu par la force de l'inertie que ces Chambres subsistent. Serait-ce l'indice d'une mentalité profondément conservatrice? C'est un des paradoxes de la société américaine. Elle subit des transformations ultra rapides, la technologie révolutionne les modes de vie, l'urbanisation pose des problèmes quasi insolubles et d'autre part les institutions politiques des Etats retardent. Le processus d'adaptation est très lent. Dans beaucoup de cas, elles constituent un cadre désuet, totalement inadéquat pour une société moderne.

Par ailleurs, il y a toujours ce réflexe traditionnel de défense contre l'exécutif, ce souci légitime de contrepoids. Même lorsque l'organe est devenu inopérant, il reste l'espoir de le réanimer. ${ }^{22}$ Ceci explique la multitude de réformes partielles proposées ou opérées, demi-mesures qui contribuent à retarder l'abolition pure et simple. On propose de réduire le nombre de sénateurs, d'allonger la durée de leur mandat; on suggère de fixer plus haut ou plus bas l'âge minimum des candidats sénateurs, d'attirer des

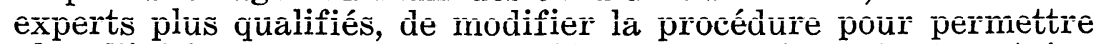
plus d'initiative, etc... Dans les Etats australiens, dont certains ont connu des majorités travaillistes, l'on s'est heurté à la même résistance passive, même si les travaillistes sont monocaméralistes par tradition. Cette institution ne gêne guère leur politique. A cela s'ajoute, semble-t-il, une sorte de mimétisme conservateur, le sénat américain et australien restant un modèle constant pour les Etats membres, quelles que soient les différences profondes qui les distinguent.

Quant au Conseil législatif de Québec, il constitue un cas particulier tant au Canada que par rapport aux sénats régionaux précités. Son abolition a été tardive si on la compare avec le sort des autres Chambres hautes provinciales au Canada; elle est hâtive par rapport aux Sénats des Etats d'Australie et des EtatsUnis. La principale raison réside dans le fait que le Conseil législatif n'était pas élu, ses membres étant pratiquement nommés à vie. C'était une sorte de petite Chambre des Lords ayant gardé intacts tous ses pouvoirs, même si elle avait de plus en plus renoncé à les exercer. Dans le passé, la force de cette Chambre s'explique avant tout parce qu'elle représentait et défendait des couches sociales supérieures, différant assez substantielle-

${ }^{22}$ De nos jours, on a un peu tendance à sous-estimer le poids du passé dans la formation de la culture politique. Locke, Montesquieu, Jefferson constituent encore, dans une certaine mesure, des composantes importantes de la psychologie sociale et politique américaine, quelles que soient les interprétations données à leurs œuvres. 
ment de celles siégeant à la Chambre élue. ${ }^{23}$ Les Sénats locaux américains et australiens avaient, eux, plutôt tendance à représenter une clientèle fort peu différente par rapport aux Chambres basses. On les accusera donc surtout de double emploi. Au Québec, les éléments sociaux privilégiés, majoritairement représentés au Conseil législatif ont finalement compris que cette institution était devenue une arme archaïque, d'une efficacité douteuse à cause de son impopularité croissante. A première vue, l'abolition du Conseil législatif n'a donc rien changé au rapport des forces en présence sur l'échiquier politique québécois. Car, tout comme aux échecs, dans les moments décisifs, une pièce complètement isolée n'a pas plus d'importance qu'une pièce perdue. Cette abolition est à la fois un signe de temps nouveaux et un symbole. Et ceci ne constitue pas un élément négligeable, surtout quand on considère l'importance des facteurs psychologiques dans la vie politique du peuple québécois. A ce point de vue, l'abolition du Conseil législatif de Québec ne risque donc pas d'influencer l'évolution du bicaméralisme régional en Australie et aux EtatsUnis, parce qu'il constitue un cas trop particulier.

23 Voir notamment le chapitre II, "Composition du Conseil Législatif" et la partie consacrée au comportement politique et social, dans E. Orban, Le Conseil Législatif de Québec (Desclée de Brouwer, Bruges, Paris, et Bellarmin, Montréal, 1967). 